PROCEEDINGS OF THE

AMERICAN MATHEMATICAL SOCIETY

Volume 135, Number 9, September 2007, Pages 2967-2973

S 0002-9939(07)08933-2

Article electronically published on May 8, 2007

\title{
UNPROVABILITY OF SHARP VERSIONS OF FRIEDMAN'S SINE-PRINCIPLE
}

\author{
ANDREY BOVYKIN
}

(Communicated by Julia Knight)

\begin{abstract}
For every $n \geq 1$ and every function $F$ of one argument, we introduce the statement $\mathrm{SP}_{F}^{n}$ : "for all $m$, there is $N$ such that for any set $A=$ $\left\{a_{1}, a_{2}, \ldots, a_{N}\right\}$ of rational numbers, there is $H \subseteq A$ of size $m$ such that for any two $n$-element subsets $a_{i_{1}}<a_{i_{2}}<\cdots<a_{i_{n}}$ and $a_{i_{1}}<a_{k_{2}}<\cdots<a_{k_{n}}$ in $H$, we have

$$
\left|\sin \left(a_{i_{1}} \cdot a_{i_{2}} \cdots a_{i_{n}}\right)-\sin \left(a_{i_{1}} \cdot a_{k_{2}} \cdots a_{k_{n}}\right)\right|<F\left(i_{1}\right) " .
$$

We prove that for $n \geq 2$ and any function $F(x)$ eventually dominated by $\left(\frac{2}{3}\right)^{\log (n-1)}(x)$, the principle $\mathrm{SP}_{F}^{n+1}$ is not provable in $I \Sigma_{n}$. In particular, the statement $\forall n \mathrm{SP}_{\left(\frac{2}{3}\right)^{\log (n-1)}}^{n}$ is not provable in Peano Arithmetic. In dimension

2 , the result is: $I \Sigma_{1}$ does not prove $\mathrm{SP}_{F}^{2}$, where $F(x)=\left(\frac{2}{3}\right) \sqrt[A^{-1}(x)]{x}$ and $A^{-1}$ is the inverse of the Ackermann function.
\end{abstract}

The study of unprovability of arithmetical statements started in the late 1970s with the invention of the method of indicators and subsequent extraction (in [10]) of a first neat unprovable statement, the Paris-Harrington Principle PH: "given natural numbers $n, k, c$, there is large enough $N$ such that for any colouring of $n$ subsets of the set $\{1,2, \ldots, N\}$ into $c$ colours, there is a subset $H$ of size at least $k$ such that our colouring is constant on $n$-subsets of $H$ and the minimal element of $H$ lies below the number $|H|$ ". The first unprovability proofs were model-theoretic, or, as in [10, finitary combinatorial approximations of model-theoretic constructions. Later, several combinatorial reformulations of $\mathrm{PH}$ were obtained, and an alternative approach to unprovability of $\mathrm{PH}$, using ordinals, was developed in [6].

Here is a reformulation of $\mathrm{PH}$ that will be relevant throughout this paper, due to Kanamori and McAloon [5. We call a function $f$ on $n$-subsets of natural numbers regressive if $f\left(x_{1}, x_{2}, \ldots, x_{n}\right) \leq x_{1}$ for all $n$-subsets $x_{1}<x_{2}<\cdots<x_{n}$ in the domain of $f$. Now, KM is the statement "for any $k, n$ there is $N$ such that for any regressive $f$ defined on $n$-subsets of $\{1,2, \ldots, N\}$, there is a subset $H$ of size $k$ such that for any $i_{1}<i_{2}<\cdots<i_{n}$ and $i_{1}<j_{2}<\cdots<j_{n}$ in $H, f\left(i_{1}, i_{2}, \ldots, i_{n}\right)=f\left(i_{1}, j_{2}, \ldots, j_{n}\right) "$. This statement is a finite version of the Regressive Ramsey theorem.

Received by the editors June 7, 2006.

2000 Mathematics Subject Classification. Primary 03F30, 03F99; Secondary 05D10.

Key words and phrases. Unprovable combinatorial statements, irrationality measure of $\pi$, dynamical system, Paris-Harrington Principle, Kanamori-McAloon Principle.

(C)2007 American Mathematical Society Reverts to public domain 28 years from publication 
In this paper we encode such ramseyan statements into a shape that does not mention quantification over all possible colourings, using chaotic behaviour of $\sin (a \cdot n)$, where $a$ is a fixed rational number.

In 2002, H. Friedman proposed the following. Let $n \gg k$ and $x_{1}, \ldots, x_{n}$ be rational numbers. There exist $p_{1}<\ldots<p_{k+2}$ such that

$$
\begin{gathered}
\left|\sin \left(x_{p_{1}} x_{p_{2}} \ldots x_{p_{k}}\right)-\sin \left(x_{p_{1}} x_{p_{3}} \ldots x_{p_{k+1}}\right)\right|<4^{-p_{1}} \\
\left|\sin \left(x_{p_{2}} x_{p_{3}} \ldots x_{p_{k+1}}\right)-\sin \left(x_{p_{2}} x_{p_{4}} \ldots x_{p_{k+2}}\right)\right|<4^{-p_{2}} .
\end{gathered}
$$

The above statement is provable in ACA but not in Peano arithmetic. The best $n=n(k)$ is $\varepsilon_{0}$-recursive but eventually dominates each $<\varepsilon_{0}$-recursive function.

Our note provides proofs of (sharp versions of) statements proposed by Friedman. We substitute the function $4^{-x}$ by some slower functions that still make the statement unprovable and provide refined $I \Sigma_{n}$-versions of the principle.

Definition 1. For every $n \in \mathbb{N}$ and any nonincreasing function $F$, let $S_{F}(n)$ be the following statement in the language of second-order arithmetic: "for every infinite increasing sequence $\left\{a_{1}, a_{2}, \ldots\right\}$ of rational numbers, there is an infinite $H \subset \mathbb{N}$ such that for any $i_{1}<i_{2}<\cdots<i_{n}<i_{n+1}$ in $H$,

$$
\left|\sin \left(a_{i_{1}} \cdot a_{i_{2}} \cdots a_{i_{n}}\right)-\sin \left(a_{i_{1}} \cdot a_{i_{3}} \cdots a_{i_{n+1}}\right)\right|<F\left(i_{1}\right) " .
$$

For every $n \in \mathbb{N}$ and any nonincreasing function $F$, let $\mathrm{SP}_{F}^{n}$ (the sine-principle for $F$ in dimension $n$ ) be the following statement in the language of first-order arithmetic: "for all $m$, there is $N$ such that for any set $A=\left\{a_{1}, a_{2}, \ldots, a_{N}\right\}$ of rational numbers, there is $H \subseteq A$ of size $m$ such that for any two $n$-element subsets $a_{i_{1}}<a_{i_{2}}<\cdots<a_{i_{n}}$ and $a_{i_{1}}<a_{k_{2}}<\cdots a_{k_{n}}$ in $H$, we have $\mid \sin \left(a_{i_{1}} \cdot a_{i_{2}} \cdots a_{i_{n}}\right)-$ $\sin \left(a_{i_{1}} \cdot a_{k_{2}} \cdots a_{k_{n}}\right) \mid<F\left(i_{1}\right) "$. The principle $\mathrm{SP}_{F}$ is defined as $\forall n \operatorname{SP}_{F}^{n}$.

To show that these principles are consistent (and provable in strong theories), notice that for any $n \in \mathbb{N}$ and any function $F$ such that $F(n) \rightarrow_{n \rightarrow \infty} 0$, we have: $\mathrm{RT}^{n} \rightarrow S_{F}(n+1) \rightarrow \mathrm{SP}_{F}^{n+1}$. Consider an infinite increasing sequence of rational numbers $A=\left\{a_{1}, a_{2}, \ldots\right\}$. Suppose we have already defined a set of indices $\left\{i_{1}, i_{2}, \ldots, i_{\ell-1}\right\}$ and an infinite set $A_{\ell}$ such that $\min A_{\ell}>a_{i_{\ell-1}}$, and for any $k<\ell$ and any two $n$-subsets $x_{1}<x_{2}<\cdots<x_{n}$ and $y_{1}<y_{2}<\cdots<y_{n}$ in $A_{\ell}$, we have $\left|\sin \left(a_{i_{k}} \cdot x_{1} \cdot \ldots \cdot x_{n}\right)-\sin \left(a_{i_{k}} \cdot y_{1} \cdot \ldots \cdot y_{n}\right)\right|<F\left(i_{k}\right)$. Define $i_{\ell}$ to be the index of min $A_{\ell}$. Partition the unit circle $S^{1}$ into $c=\left[\frac{2 \pi+1}{F\left(i_{\ell}\right)}\right]$ neighbourhoods of length less than $F\left(i_{\ell}\right)$ and define a colouring $f:\left[A_{\ell}\right]^{n} \rightarrow c$ as follows: $f\left(x_{1}, \ldots, x_{n}\right)$ is the neighbourhood of $e^{i \cdot a_{\ell} \cdot x_{1} \cdot \ldots \cdot x_{n}}$. By $\mathrm{RT}^{n}$, there is an infinite set $X \subseteq A_{\ell}$, homogeneous for $f$. Put $A_{\ell+1}=X$. Clearly, the set $\left\{a_{i_{\ell}} \mid \ell \in \mathbb{N}\right\}$ is as required.

Here is our main theorem.

Theorem 1. For every $n \geq 2, \mathrm{SP}_{F}^{n+1}$ is not provable in $I \Sigma_{n}$, where $F(x)=$ $\left(\frac{2}{3}\right)^{\log ^{(n-1)}(x)}$ and $\log ^{(n-1)}$ is the binary logarithm function iterated $(n-1)$ times. In dimension 2, the result is: $I \Sigma_{1}$ does not prove $\mathrm{SP}_{F}^{2}$, where $F(x)=\left(\frac{2}{3}\right)^{A^{-1}(x)} \sqrt{x}$ and $A^{-1}$ is the inverse of the Ackermann function.

\section{Proof of Theorem 1}

We shall prove Theorem 1 by giving an elementary proof that for any $n \geq 2$, we have: $\mathrm{SP}_{F}^{n+1}$ implies $\mathrm{KM}_{\log ^{(n-1)}}^{n+1}$, the $\log ^{(n-1)}$-version of the Kanamori-McAloon Principle, which is known to be unprovable in $I \Sigma_{n}$. 
Throughout the proof, we denote the set $\{1,2, \ldots, N\}$ by $N$ and the set of $n$-subsets of $N$ by $[N]^{n}$. For natural numbers $m, n, N$, we say that a function $f:[N]^{n} \rightarrow N$ is $\log ^{(m)}$-regressive if $f\left(x_{1}, x_{2}, \ldots, x_{n}\right) \leq \log ^{(m)}\left(x_{1}\right)$ for any $n$-subset $x_{1}<x_{2}<\cdots<x_{n}$ of $N$. The principle $\mathrm{KM}_{\log (m)}^{n}$ says: "for all $k$, there is $N$ such that whenever $f:[N]^{n} \rightarrow N$ is $\log ^{(m)}$-regressive, there is a min-homogeneous subset $H \subset N$ of size $k$, i.e., for any two $n$-element subsets of $H$ of the form $i_{1}<$ $i_{2}<\cdots<i_{n}$ and $i_{1}<j_{2}<\cdots<j_{n}$, we have $f\left(i_{1}, i_{2}, \ldots, i_{n}\right)=f\left(i_{1}, j_{2}, \ldots, j_{n}\right)$ " .

We shall use the following refined threshold classification that was obtained recently in the work of Weiermann, Carlucci and Lee [2]: for any $n \geq 2, k \geq 0$, if $k \leq n-1$, then $\mathrm{KM}_{\log ^{(k)}}^{n+1}$ is $I \Sigma_{n^{-}}$unprovable, otherwise $\mathrm{KM}_{\log (k)}^{n+1}$ is provable in $I \Delta_{0}+\exp$. For $n=1$ the result is [7]: $I \Sigma_{1}$ does not prove $\mathrm{KM}_{f}^{(2)}$, where $f(x)=x^{\frac{1}{A^{-1}(x)}}$, but for every $m \in \mathbb{N}, I \Sigma_{1}$ proves $\mathrm{KM}_{f_{m}}^{(2)}$, where $f_{m}(x)=x^{\frac{1}{F_{m}^{-1}(x)}}$ and $\left\{F_{m}\right\}_{m \in \mathbb{N}}$ is Grzegorczyk's hierarchy of primitive recursive functions. The model-theoretic treatment of threshold results for KM and related statements can be found in [1].

Lemma 2 (Every function can be approximated by sine on a subset). For any $\varepsilon>0$ and any dimension $n$, any number $K$ and any function $g:[K]^{n} \rightarrow[-1,1]$, there is a set of rational numbers $A=\left\{a_{1}, a_{2}, \ldots, a_{K}\right\}$ such that for any $i_{1}<i_{2}<$ $\cdots<i_{n} \leq K$, we have

$$
\left|g\left(i_{1}, i_{2}, \ldots, i_{n}\right)-\sin \left(a_{i_{1}} \cdot a_{i_{2}} \cdots a_{i_{n}}\right)\right|<\varepsilon .
$$

Let us first show how Lemma 2 implies our theorem.

Proof of Theorem 1. Let us show that for any $n \geq 2, I \Sigma_{1}$ proves that $\mathrm{SP}_{F}^{n+1}$ implies $\mathrm{KM}_{\log (n-1)}^{n+1}$, hence $\mathrm{SP}_{F}^{n+1}$ is unprovable in $I \Sigma_{n}$.

Fix $m>n$ and let $N$ be such that among any $N$ rational numbers $\left\{a_{1}, \ldots, a_{N}\right\}$, there is a subset $H$ of size $m$ such that for any two $(n+1)$-subsets $a_{i_{1}}<a_{i_{2}}<$ $\cdots<a_{i_{n+1}}$ and $a_{i_{1}}<a_{k_{2}}<\cdots<a_{k_{n+1}}$ in $H$, we have

$$
\left|\sin \left(a_{i_{1}} \cdot a_{i_{2}} \cdots a_{i_{n+1}}\right)-\sin \left(a_{i_{1}} \cdot a_{k_{2}} \cdots a_{k_{n+1}}\right)\right|<F\left(i_{1}\right) \text {. }
$$

Let us define a mapping $h:\{1,2, \ldots, N\} \rightarrow[-1,1]$ as follows. Let $\varepsilon=\frac{2-\sum_{i=1}^{N}\left(\frac{2}{3}\right)^{i}}{2 N}$. Put $h(1)=-1$ and for every $i<N, h(i+1)=h(i)+2 \varepsilon+\left(\frac{2}{3}\right)^{i}$. Clearly, $h(i)$ differs from images of other points by at least $\left(\frac{2}{3}\right)^{i}+2 \varepsilon$.

Let $f:[N]^{n+1} \rightarrow N$ be an arbitrary $\log ^{(n-1)}$-regressive function and define $g\left(i_{1}, i_{2}, \ldots, i_{n+1}\right)=h\left(f\left(i_{1}, i_{2}, \ldots, i_{n+1}\right)\right)$. So, the point $g\left(i_{1}, i_{2}, \ldots, i_{n+1}\right)$ differs from neighbouring images of $g$ by at least $\left(\frac{2}{3}\right)^{\log ^{(n-1)}\left(i_{1}\right)}+2 \varepsilon$.

Using Lemma 2, choose a subset $A=\left\{a_{1}, a_{2}, \ldots, a_{N}\right\}$ of rational numbers such that for all $i_{1}<i_{2}<\cdots<i_{n}<N$,

$$
\left|g\left(i_{1}, i_{2}, \ldots, i_{n+1}\right)-\sin \left(a_{i_{1}} \cdot a_{i_{2}} \cdots a_{i_{n+1}}\right)\right|<\varepsilon .
$$

Applying $\mathrm{SP}_{F}^{n+1}$ to this set, extract a subset $H \subseteq\{1,2, \ldots, N\}$ of size $m$ such that on $H$,

$$
\left|\sin \left(a_{i_{1}} \cdot a_{i_{2}} \cdots a_{i_{n+1}}\right)-\sin \left(a_{i_{1}} \cdot a_{k_{2}} \cdots a_{k_{n+1}}\right)\right|<F\left(i_{1}\right) .
$$


Let us notice that $H$ is exactly the min-homogeneous set we are seeking. Indeed, on $H$,

$$
\begin{gathered}
\left|g\left(i_{1}, i_{2}, \ldots, i_{n+1}\right)-g\left(i_{1}, k_{2}, \ldots, k_{n+1}\right)\right| \leq\left|g\left(i_{1}, i_{2}, \ldots, i_{n+1}\right)-\sin \left(a_{i_{1}} \cdot a_{i_{2}} \cdots a_{i_{n+1}}\right)\right| \\
+\left|\sin \left(a_{i_{1}} \cdot a_{i_{2}} \cdots a_{i_{n+1}}\right)-\sin \left(a_{i_{1}} \cdot a_{k_{2}} \cdots a_{k_{n+1}}\right)\right| \\
+\left|g\left(i_{1}, k_{2}, \ldots, k_{n+1}\right)-\sin \left(a_{i_{1}} \cdot a_{k_{2}} \cdots a_{k_{n+1}}\right)\right|<\varepsilon+\varepsilon+\left(\frac{2}{3}\right)^{\log ^{(n-1)}\left(i_{1}\right)}
\end{gathered}
$$

Now, since different values of $g$ would differ by at least $\left(\frac{2}{3}\right)^{\log ^{(n-1)}\left(i_{1}\right)}+2 \varepsilon$, we conclude that $g\left(i_{1}, i_{2}, \ldots, i_{n}\right)=g\left(i_{1}, k_{2}, \ldots, k_{n}\right)$.

\section{Proof of Lemma 2}

We shall use the Rhin-Viola Theorem on irrationality measure of $\pi$ from [1]: for all $n, k \in \mathbb{N},|n-\pi k|>n^{-10}$.

Lemma 3. Let $x \in \mathbb{R}, \delta>0, \frac{a}{b} \in \mathbb{Q} \backslash\{0\}$. Then the finite set

$$
\left\{e^{i \cdot\left(x+\frac{a}{b} \cdot k\right)} \mid k=0,1,2, \ldots,\left[\frac{(2 \pi)^{12}}{\delta^{11}} \cdot a^{10} b\right]+1\right\}
$$

has a nonempty intersection with any arc of length $\delta$ on the unit circle.

Proof. Let us first show that there is $k_{0} \leq \frac{2 \pi}{\delta}+1$ such that for some $m \in \mathbb{N}$,

$$
\delta>\left|\frac{a}{b} \cdot k_{0}-\pi m\right|
$$

Divide $S^{1}$ into $\left[\frac{2 \pi}{\delta}\right]+1$ arcs of length $<\delta$ and notice that by pigeonhole principle, there are $k_{1}<k_{2} \leq\left[\frac{2 \pi}{\delta}\right]+2$ such that

$$
\left|e^{i\left(x+\frac{a}{b} k_{1}\right)}-e^{i\left(x+\frac{a}{b} k_{2}\right)}\right|<\delta
$$

hence, $\left|e^{i \frac{a}{b}\left(k_{2}-k_{1}\right)}-1\right|<\delta$ as desired. Set $k_{0}=k_{2}-k_{1}$.

By the Rhin-Viola Theorem [11, for all $a, b, m, k \in \mathbb{N}$,

$$
\begin{gathered}
|a k-\pi m b|>(a k)^{-10}, \text { so } \\
\delta>\left|\frac{a}{b} \cdot k_{0}-\pi m\right|>\frac{1}{\left(a k_{0}\right)^{10} \cdot b} .
\end{gathered}
$$

Now, divide $S^{1}$ into $\left[2 \pi\left(a k_{0}\right)^{10} b\right]+1$ arcs of length $<\frac{1}{\left(a k_{0}\right)^{10} b}$ and notice that

$$
k_{0} \cdot\left(a k_{0}\right)^{10} \cdot b \cdot 2 \pi \leq(2 \pi)^{12} \cdot\left(\frac{1}{\delta}\right)^{11} \cdot a^{10} b
$$

steps of $e^{i\left(x+k_{0} \frac{a}{b} j\right)}$ for varying $j$ go all way round $S^{1}$ visiting every $\delta$-neighbourhood.

In order to prove Lemma 2, we shall first construct a sequence $\left\{r_{1}, r_{2}, \ldots, r_{K}\right\}$ of irrational numbers of the form $r_{i}=\sqrt[n-1]{\pi} \cdot \frac{a_{i}}{b_{i}}$ such that

$$
\left|\sin \left(r_{i_{1}} \cdot r_{i_{2}} \cdot \ldots \cdot r_{i_{n}}\right)-g\left(i_{1}, i_{2}, \ldots, i_{n}\right)\right|<\frac{\varepsilon}{2}
$$

and then find rational numbers $\left\{a_{1}, a_{2}, \ldots, a_{K}\right\}$ such that $a_{i}$ is so close to $r_{i}$ that

$$
\left|\sin \left(r_{i_{1}} \cdot r_{i_{2}} \cdot \ldots \cdot r_{i_{n}}\right)-\sin \left(a_{i_{1}} \cdot a_{i_{2}} \cdot \ldots \cdot a_{i_{n}}\right)\right|<\frac{\varepsilon}{2} .
$$


Let us start off by choosing any $n$ numbers $r_{1}<r_{2}<\cdots<r_{n}$ of the desired form and such that $\left|\sin \left(r_{1} \cdot r_{2} \cdot \ldots \cdot r_{n}\right)-g(1,2, \ldots, n)\right|<\frac{\varepsilon}{2}$.

Lemma 4. Suppose for $m \geq n$, we have constructed $m$ numbers $r_{1}, r_{2}, \ldots, r_{m}$ of the form $r_{i}=\sqrt[n-1]{\pi} \cdot \frac{a_{i}}{b_{i}}$, where $a_{i}, b_{i} \in \mathbb{N}$ and for every $i_{1}<\cdots<i_{n-1} \leq m$ we have fixed a number $g\left(i_{1}, \ldots, i_{n-1}, m+1\right) \in[-1,1]$. Enumerate all products $r_{i_{1}} \cdot r_{i_{2}} \cdot \ldots \cdot r_{i_{n-1}}$ as $\left\{R_{i}\right\}_{i=1}^{C_{m}^{n-1}}$ which clearly have the form $R_{i}=\pi \cdot \frac{p_{i}}{q_{i}}$. Define a function $f(i)$ as follows: $f(1)=\frac{2 q_{1}}{p_{1}}$,

$$
f(j+1)=f(j)+\frac{2^{34} \cdot \pi^{12} R_{j+1}^{11}}{\varepsilon^{11}} \cdot\left(q_{1} q_{2} \ldots q_{j}\right)^{11} .
$$

Then there is $r_{m+1}=\sqrt[n-1]{\pi} \cdot \frac{a_{m+1}}{b_{m+1}}$ such that for every $i_{1}<\cdots<i_{n-1} \leq m$,

$$
\left|\sin \left(r_{i_{1}} \cdot r_{i_{2}} \cdot \ldots \cdot r_{i_{n-1}} \cdot r_{m+1}\right)-g\left(i_{1}, i_{2}, \ldots, i_{n-1}, m+1\right)\right|<\frac{\varepsilon}{2}
$$

and $a_{m+1}<\frac{2 R_{C_{m}^{n-1}}}{\varepsilon} \cdot \frac{f\left(C_{m}^{n-1}\right)}{n-\sqrt[1]{\pi}}, b_{m+1}<\frac{2 R_{C_{m}^{n-1}}}{\varepsilon}$.

Proof. Enumerate the corresponding numbers $g\left(i_{1}, i_{2}, \ldots, i_{n-1}, m+1\right)$ as $\left\{h_{j}\right\}_{j=1}^{C_{m}^{n-1}}$.

Let $x_{1} \in \mathbb{R}$ be such that $\sin \left(R_{1} \cdot x_{1}\right)=h_{1}$. The number $x_{1}$ can clearly be chosen below $f(1)$, and any number of the form $x_{1}+\frac{2 q_{1}}{p_{1}} \cdot k(k \in \mathbb{Z})$ satisfies this equality.

Suppose for $j \leq C_{m}^{n-1}$, we have built a point $x_{j}$ below $f(j)$ such that for all $i \leq j$,

$$
\left|\sin \left(R_{i} \cdot x_{j}\right)-h_{i}\right|<\frac{\varepsilon}{2} .
$$

Then such is every point of the form $x_{j}+2 \cdot q_{1} q_{2} \ldots q_{j} \cdot k(k \in \mathbb{Z})$, because $2 q_{1} q_{2} \ldots q_{j}$ is a common period of $\sin \left(R_{i} x\right)$ for $i \leq j$ (but not the least common period, which is $\left.2 \mathrm{LCF}\left(\frac{q_{1}}{p_{1}}, \ldots, \frac{q_{j}}{p_{j}}\right)\right)$.

By Lemma 3, there is

$$
k_{j} \leq \frac{2^{33} \cdot \pi^{12} \cdot R_{j+1}^{11}}{\varepsilon^{11}} \cdot\left(q_{1} q_{2} \ldots q_{j}\right)^{10}
$$

such that

$$
x_{j+1}=x_{j}+2 \cdot\left(q_{1} q_{2} \ldots q_{j}\right) \cdot k_{j}
$$

belongs to an $\frac{\varepsilon}{2 R_{j+1}}$-neighbourhood of a point $y$ such that $\sin \left(R_{j+1} \cdot y\right)=h_{j+1}$. Clearly then $x_{j+1}$ also satisfies the new inequality

$$
\left|\sin \left(R_{j+1} \cdot x_{j+1}\right)-h_{j+1}\right|<\frac{\varepsilon}{2} .
$$

We still keep track of the size of $x_{j+1}$ :

$$
x_{j+1}=x_{j}+\frac{2^{34} \cdot \pi^{12} R_{j+1}^{11}}{\varepsilon^{11}} \cdot\left(q_{1} q_{2} \ldots q_{j}\right)^{11} \leq f(j+1) .
$$

Now, finally, for $j=C_{m}^{n-1}$ and $\delta=\frac{1}{2} \min \left\{\frac{\varepsilon}{R_{1}}, \ldots, \frac{\varepsilon}{R_{j}}\right\}=\frac{\varepsilon}{2 R_{j}}$, let us choose the point $r_{m+1}$ within a $\delta$-neighbourhood 11 of $x_{j}$ to make sure that $\left|\sin \left(R_{i} \cdot r_{m+1}\right)-h_{i}\right|<$ $\frac{\varepsilon}{2}$ for all $i \leq C_{m}^{n-1}$.

\footnotetext{
${ }^{1}$ We can assume $R_{C_{m}^{n-1}}$ is the largest one in our enumeration.
} 
Since in every $\delta$-neighbourhood below $A$ there is a rational number $\frac{a}{b}$ such that $b<\frac{1}{\delta}$ and $a<\frac{A}{\delta}$, we can pick

$$
b_{m+1}<\frac{2 R_{C_{m}^{n-1}}}{\varepsilon} \text { and } a_{m+1}<\frac{2 R_{C_{m}^{n-1}}}{\varepsilon} \cdot \frac{f\left(C_{m}^{n-1}\right)}{\sqrt[n-1]{\pi}} .
$$

Let us now complete the proof of Lemma 2. Notice that in order to build $r_{m+1}$ we made $k_{1}$ steps with pace $2 q_{1}$, then $k_{2}$ steps with pace $2 q_{1} q_{2}$, etc., then finally $k_{C_{m}^{n-1}}$ steps with pace $2 q_{1} \ldots q_{C_{m}^{n-1}}$. Now it is easy to explicitly write a number $\delta_{m+1}$ such that for any numbers $x_{1}, \ldots, x_{m}, x_{m+1}$ such that $x_{i}$ is within the $\delta_{m+1^{-}}$ neighbourhood of $r_{i}$, we have

$$
\left|\sin \left(x_{i_{1}} \cdot \ldots \cdot x_{i_{n-1}} \cdot x_{m+1}\right)-\sin \left(r_{i_{1}} \cdot \ldots \cdot r_{i_{n-1}} \cdot r_{m+1}\right)\right|<\frac{\varepsilon}{2}
$$

for any $i_{1}<\cdots<i_{n-1}<m+1$. Set $\delta=\min _{i \leq K} \delta_{i}$ and choose our set $\left\{a_{i}\right\}_{i=1}^{K}$ so that $a_{i}$ is within the $\delta$-neighbourhood of $r_{i}$.

Note that we did not use the actual value $n^{-10}$ from the Rhin-Viola Theorem but only existence of an effective bound. So, we could well quote the early effective bound by Feldman [4] or a theorem of Mahler [9]: $|\pi k-n|>\frac{1}{k^{41}}$.

Notice also that by construction of Lemma 4 , the sine-principle is equivalent to the statement in which the quantifier "for all sequences $\left\{a_{i}\right\}_{i=1}^{N}$ of rational numbers" is replaced by a bounded quantifier "for all sequences $\left\{a_{i}\right\}_{i=1}^{N}$ of rational numbers where the numerator and denominator of $a_{i}$ are bounded".

There are possibilities to extend Theorem 1 in a nonsuperficial way. The first one is about sine of natural arguments. I suggest that the following principle is still unprovable in $I \Sigma_{n}$ : "for all $m$, there is $N$ such that for any set $A=\left\{a_{1}, a_{2}, \ldots, a_{N}\right\}$ of natural numbers, there is $H \subseteq A$ of size $m$ such that for any two $(n+1)$-element subsets $a_{i_{1}}<a_{i_{2}}<\cdots<a_{i_{n+1}}$ and $a_{i_{1}}<a_{k_{2}}<\cdots<a_{k_{n+1}}$ in $H$, we have

$$
\left|\sin \left(a_{i_{1}} \cdot a_{i_{2}} \cdots a_{i_{n+1}}\right)-\sin \left(a_{i_{1}} \cdot a_{k_{2}} \cdots a_{k_{n+1}}\right)\right|<\left(\frac{2}{3}\right)^{\log (n-1)\left(i_{1}\right)} " .
$$

It was suggested by the referee that this conjecture may be tackled by applying a result of $\mathrm{H}$. Weyl.

The second conjecture is about zeta-function. The final theorem may look like: for any $n \geq 2$, the statement "for all $m$, there is $N$ such that for any set $A=$ $\left\{a_{1}, a_{2}, \ldots, a_{N}\right\}$ of rational complex numbers, there is $H \subseteq A$ of size $m$ such that for any two $n$-element subsets $a_{i_{1}}<a_{i_{2}}<\cdots<a_{i_{n}}$ and $a_{i_{1}}<a_{k_{2}}<\cdots<a_{k_{n}}$ in $H$, we have $\left|\zeta\left(a_{i_{1}} \cdot a_{i_{2}} \cdots a_{i_{n}}\right)-\zeta\left(a_{i_{1}} \cdot a_{k_{2}} \cdots a_{k_{n}}\right)\right|<\frac{1}{i_{1}}$ " is unprovable in $I \Sigma_{n-1}$. In particular, for $n=2$, it is a simple number-theoretic statement that does not have an 'elementary' proof. To do this we need an analogue of Lemma 2 for the zetafunction. This can probably be done by using existing results on value distribution of the zeta-function on vertical lines.

Clearly, every function that satisfies Lemma 2 gives us an independence result of a similar shape. So, an interesting investigation would be to catalogue a few other examples of this kind and convert them into shapes that are interesting in the corresponding mathematical disciplines.

Recently, A.Weiermann found another proof of Lemma 2, using an effective version of Kronecker's result on simultaneous diophantine approximation. This led him to formulate an infinite family of unprovable statements that use the function $\left\{a_{i_{1}} \cdot \ldots \cdot a_{i_{n}}\right\}$ (where $\{x\}$ is the fractional part of $x$ ) in place of $\sin \left(a_{i_{1}} \cdot \ldots \cdot a_{i_{n}}\right)$. 
One last idea is to extend the principle to other dynamical systems (or classes of dynamical systems) by encoding ramsey-theoretic phenomena as in Lemma 2.

I would like to thank Professor Grigori Mints for posing the original problem to me, Dr. Maxim Vsemirnov for referring me to the Rhin-Viola Theorem and the anonymous referee for suggesting future ways to solve my conjectures.

\section{REFERENCES}

[1] Bovykin, A. (2005). Model-theoretic treatment of threshold results for PH. Manuscript, downloadable from http://logic.pdmi.ras.ru/ andrey/research.html.

[2] Carlucci L., Lee G., Weiermann, A. (2005). Classifying the phase transition threshold for regressive Ramsey functions. Submitted.

[3] Friedman, H. (2002). A posting in the internet forum FOM. June 8, 2002. http://www.cs.nyu.edu/pipermail/fom/2002-June/005584.html

[4] Feldman, N.I. (1951). The approximation of certain transcendental numbers. I. Approximation of logarithms of algebraic numbers. (in Russian) Izvestiya Akademii Nauk SSSR, Mathematics series, 15, pp. 53-74. MR0039768(12:595d)

[5] Kanamori, A., McAloon, K. (1987). On Gödel incompleteness and finite combinatorics. Annals of Pure and Applied Logic, 33, pp. 23-41. MR0870685 (88i:03095)

[6] Ketonen, J., Solovay, R. (1981). Rapidly growing Ramsey Functions. Annals of Mathematics (ser 2) 113, pp. 267-314. MR0607894 (84c:03100)

[7] Kojman, M., Lee, G., Omri, E., Weiermann, A. (2005). Sharp thresholds for the phase transition between primitive recursive and ackermannian Ramsey numbers. Submitted.

[8] Lee, G. (2005). Phase transitions in axiomatic thought. Ph.D. Thesis, University of Münster.

[9] Mahler, K. (1953). On the approximation of $\pi$. Nederl. Akad. Wetensch. Proc. Ser. A. 56 pp. 30-42. MR0054660 (14:957a)

[10] Paris, J., Harrington, L. (1977). A mathematical incompleteness in Peano arithmetic. Handbook for Mathematical Logic, North-Holland.

[11] Rhin, G. Viola, C. (2001). The group structure of $\zeta(3)$. Acta Arithmetica, 97 (3), pp. 269-293. MR1826005 (2002b:11098)

Steklov Mathematical Institute, Fontanka 27, St. Petersburg, Russia; Liverpool University, Liverpool, United Kingdom

E-mail address: andrey@logic.pdmi.ras.ru 